\title{
Evaluation of Tigecycline and Minocycline Susceptibility among Clinical Isolates of Carbapenem Resistant Acinetobacter Species
}

\author{
Asna Parveen ${ }^{1}$, Pratibha Bhat ${ }^{2}$ \\ 1, 2 Department of Microbiology, Nitte (Deemed to Be University), KS Hegde \\ Medical Academy (KSHEMA), Deralakatte, Mangaluru, Karnataka, India.
}

\section{ABSTRACT}

\section{BACKGROUND}

Acinetobacter species are important infectious agents worldwide especially in healthcare settings. It has the ability to develop various resistance mechanisms to various antibiotics. We wanted to study the role of tigecycline and minocycline in the treatment of multidrug resistant Acinetobacter species.

\section{METHODS}

254 non-repetitive isolates of Acinetobacter species from various clinical samples like exudates, blood, sputum, urine were retrospectively studied. Antibiotic susceptibility testing was done by Vitek 2 compact system. Susceptibility of the carbapenem resistant isolates towards tigecycline and minocycline were analysed.

\section{RESULTS}

$205(80.7 \%)$ isolates were resistant to either of the carbapenem drugs and 49 (19.3 $\%)$ were sensitive to all the 3 carbapenems, namely imipenem, meropenem and doripenem. $54.1 \%$ isolates were sensitive to tigecycline while sensitivity towards minocycline was $40.5 \%$. The degree of sensitive concordance in the susceptibility to minocycline and tigecycline against Acinetobacter species was $31.1 \%$, which indicated fair agreement statistically. $21.1 \%$ isolates were resistant / intermediate to minocycline but sensitive to tigecycline. Only $9.4 \%$ isolates which were resistant to tigecycline were sensitive to minocycline.

\section{CONCLUSIONS}

The results of the present study have demonstrated that minocycline and tigecycline are effective against the carbapenem resistant Acinetobacter species. Tigecycline can be considered as a therapeutic agent for the treatment of multidrug resistant Acinetobacter which are otherwise difficult to inhibit using other antibiotics.

\section{KEY WORDS}

Carbapenem Resistance, Tigecycline, Minocycline, Antimicrobial Resistance
Corresponding Author: Dr. Pratibha Bhat U., Assistant Professor, Department of Microbiology, Nitte (Deemed to Be University), KS Hegde Medical Academy (KSHEMA), Deralakatte, Mangaluru - 575018, Karnataka, India.

E-mail: pratibhabhat.u@gmail.com

DOI: $10.14260 / j e m d s / 2021 / 297$

How to Cite This Article:

Parveen A, Bhat P. Evaluation of tigecycline and minocycline susceptibility among clinical isolates of carbapenem resistant acinetobacter species. J Evolution Med Dent Sci 2021;10(19):1408-1412, DOI: 10.14260/jemds/2021/297

Submission 03-01-2021,

Peer Review 11-03-2021,

Acceptance 17-03-2021,

Published 10-05-2021.

Copyright (c) 2021 Asna Parveen et al. This is an open access article distributed under Creative Commons Attribution License [Attribution 4.0 International (CC BY 4.0)] 


\section{BACKGROUND}

Acinetobacter species are non-motile, non-fermenting gram negative coccobacilli. Worldwide, they are renowned to be one of the most difficult drug resistant bugs to control and treat. They are an important cause of hospital acquired infections like ventilator associated pneumonias, blood stream infections, skin and soft tissue infections, urinary tract infections. The mortality rate can amount to about $40 \%$ in the intensive care unit settings (ICUs). ${ }^{1}$

Among the different species of Acinetobacter, A. baumannii is identified as the most significant pathogen. Reasons for this are that it can remain viable for long periods on surfaces and equipment's used in health care. It can also quickly develop resistance to the commonly used antibiotics. Acinetobacter species have great potential to cause life threatening infections in critically ill patients. Also, outbreaks of infections due to contamination of patient care equipment's in hospitals, attributable to Acinetobacter have been described. Meticulous and stringent infection control measures and precise antibiotic therapy is the need of the hour to prevent the spread of multidrug resistant Acinetobacter infections. Exact reservoir of Acinetobacter is not ascertained. Though skin carriage has been implicated as a cause of nosocomial outbreaks of infection, the rate of carriage of Acinetobacter baumannii is only $1-3 \%$. Other species of Acinetobacter are more frequently associated with skin carriage. ${ }^{1}$

Carbapenems like imipenem, meropenem and doripenem and aminoglycosides like amikacin and gentamicin are the commonly used drugs to treat serious infections caused by Acinetobacter species. However, increasing resistance to these group of antibiotics has been recorded especially in South and South-East Asian countries. ${ }^{2,3}$ Polymyxins, tigecycline, minocycline and sulbactam are some of the existing therapeutic options for carbapenem resistant Acinetobacter species. Siderophore cephalosporins like cefiderocol, a tetracycline drug namely eravacycline are some of the newer drugs in the pipeline against these drug resistant species, but they are yet to reach the bedside. ${ }^{4}$

This urges the need to study the action of the existing antimicrobials and their judicious use. This has led to an increased use of minocycline and tigecycline to treat these infections. ${ }^{5}$ Minocycline is a tetracycline with a broad spectrum of activity. It acts by inhibiting the protein synthesis in bacteria by binding to 30 s ribosome and blocking the entry of transfer RNA. Tigecycline is a glycylcycline, a tetracycline derivative antibiotic. It has a unique mechanism of action against gram negative bacteria. The $9^{\text {th }}$ position of the molecule is substituted with a large $\mathrm{N}$-alkyl glycylamido group, which helps in overcoming most of the tetracycline resistant mechanisms. ${ }^{6}$ Study of susceptibility of Acinetobacter species towards tigecycline and minocycline would help in the effective treatment and control of Acinetobacter species infections in the hospital.

\section{METHODS}

This was a retrospective study done in the microbiology laboratory of a tertiary care centre in South India. Data from March 2018 to June 2019 was collected retrospectively. Non repetitive isolates from various clinical samples like exudates, sputum, blood and urine, which were identified as Acinetobacter species by standard biochemical tests or by Vitek 2 compact system were considered. The antimicrobial susceptibility testing for these isolates were done by Vitek 2 compact system. Isolates of Acinetobacter species of which antimicrobial susceptibility testing was done by methods other than Vitek 2 system were excluded from the study.

Antibiotic susceptibility pattern of the isolates towards 3 carbapenem antibiotics namely imipenem, meropenem, doripenem were tabulated. Tigecycline and minocycline susceptibility data was also recorded. The minimum inhibitory concentration (MIC) values for the carbapenems and minocycline were interpreted as sensitive, intermediate or resistant based on the Clinical Laboratory and Standards Institute (CLSI) document, 2018.7 MIC of $<2 \mu \mathrm{g} / \mathrm{ml}$ as sensitive, $4 \mu \mathrm{g} / \mathrm{ml}$ as intermediate and $>8 \mu \mathrm{g} / \mathrm{ml}$ as resistant was considered for all the 3 carbapenems. For minocycline the interpretation (sensitive $<4 \mu \mathrm{g} / \mathrm{ml}$, intermediate $=8 \mu \mathrm{g} / \mathrm{ml}$, resistant $\geq 16 \mu \mathrm{g} / \mathrm{ml}$ ) was considered. There are no guidelines available for tigecycline breakpoints for Acinetobacter species. The common practice is to use the European committee on antimicrobial susceptibility testing (EUCAST, 2018) guidelines $^{8}$ set for Enterobacterales to interpret the tigecycline MICs of Acinetobacter species as well. The MIC was interpreted as sensitive $=1 \mu \mathrm{g} / \mathrm{ml}$; intermediate $=2 \mu \mathrm{g} / \mathrm{ml}$, resistant $>2 \mu \mathrm{g} / \mathrm{ml} .8,9$

\section{Statistical Analysis}

The collected data was tabulated in Microsoft Excel sheet and statistically analysed. Descriptive statistics such as counts and percentages were used to express qualitative data. Cohen Kappa values were used to assess concordance. Concordance means the chances that if tigecycline was sensitive, minocycline would also be sensitive or if tigecycline showed resistance, minocycline too would be the same. Kappa values of $</=0$ indicated no agreement, $0.0-0.20$ indicated none to slight agreement, $0.21-0.40$ indicated fair, $0.41-0.60$ moderate, $0.61-0.80$ substantial and $0.81-1.00$ as almost perfect agreement. ${ }^{10}$

\section{RESULTS}

A total of 254 isolates of Acinetobacter species were considered; 166 from exudates samples, 18 from blood samples, 21 urine isolates and 49 from sputum samples. Out of which, $205(80.7 \%)$ isolates were resistant to either of the carbapenem drugs as shown in Table 1, and 49 (19.3\%) were sensitive to all the 3 carbapenems.

\begin{tabular}{|ccc|}
\hline Sample & Number & Percentage \\
Exudates $(\mathrm{N}=166)$ & 141 & $84.9 \%$ \\
Blood $(\mathrm{N}=18)$ & 16 & $88.9 \%$ \\
Urine $(\mathrm{N}=21)$ & 11 & $52.3 \%$ \\
Sputum $(\mathrm{N}=49)$ & 37 & $75.5 \%$ \\
\hline Table 1. Sample Wise Distribution of Isolates \\
Resistant to Either of the Carbapenems \\
\hline
\end{tabular}

Data of tigecycline susceptibility was available for all the 205 isolates of carbapenem resistant Acinetobacter, but minocycline susceptibility data was available for only 180 isolates. $54.1 \%$ isolates were sensitive to tigecycline while 
sensitivity towards minocycline was $40.5 \%$ as shown in Table 2.

\begin{tabular}{|cccc|}
\hline Antibiotic & Sensitive & Intermediate & Resistant \\
N (\%) & N (\%) & N (\%) \\
Tigecycline ( $=205)$ & $111(54.1)$ & $70(34.1)$ & $24(11.7)$ \\
Minocycline $(\mathrm{N}=180)$ & $73(40.5)$ & $25(13.9)$ & $82(45.6)$ \\
\hline \multicolumn{4}{|c|}{ Table 2. In Vitro Susceptibility of Carbapenem Resistant } \\
Acinetobacter Species against Tigecycline and Minocycline \\
\hline
\end{tabular}

Highest resistance towards tigecycline and minocycline was noted in carbapenem resistant isolates from blood $(62.5$ $\%$ and $64.3 \%$ respectively) followed by exudates ( $47.5 \%, 58.2$ $\%)$ and least was seen in isolates from urine samples (0\%, 36.4 $\%$ ) as shown in Figure 1.

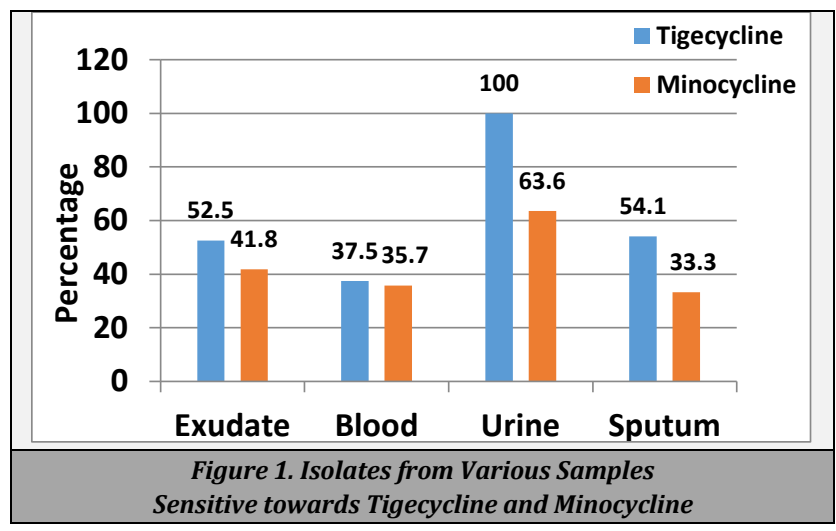

Out of the 205 isolates, $183(89.3 \%)$ were resistant to all the 3 carbapenems, and $11(5.4 \%)$ were resistant to 2 carbapenem drugs and $11(5.4 \%)$ were resistant to a single carbapenem drug as shown in Table $3 \mathrm{a}$ and $3 \mathrm{~b}$.

\begin{tabular}{|c|c|c|c|}
\hline Degree of Carbapenem Resistance & \multicolumn{2}{|c|}{ SensitiveIntermediate } & $\begin{array}{c}\text { Resistant } \\
\text { No. (\%) }\end{array}$ \\
\hline Res & 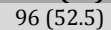 & & $22(12.2)$ \\
\hline Resis & & & 2( \\
\hline Resistant to any 1 carbapen & $8(7$ & $2(18.2)$ & \\
\hline \multicolumn{4}{|c|}{ Sensitivity towards Tigecycline in Relation to Resistance towards Carbapenems } \\
\hline Resistant to all 3 carbapenems ( $\mathrm{n}=164$ ) & $63(38.4)$ & $24(14.6)$ & $77(47)$ \\
\hline Resist: & & & \\
\hline Resistant to any 1 carbapenem $(n=7)$ & 5 (71.4) & $1(14.3)$ & $1(14.3)$ \\
\hline \multicolumn{4}{|c|}{ Sensitivity towards Minocycline in Relation to Resistance towards Carbapenems } \\
\hline \multicolumn{4}{|c|}{ Table 3.} \\
\hline
\end{tabular}

\begin{tabular}{|c|c|c|c|c|}
\hline & & \multicolumn{2}{|c|}{ Minocycline } & \multirow{2}{*}{ Tota } \\
\hline & & $\mathbf{S}$ & $\mathbf{R} / \mathbf{I}$ & \\
\hline \multirow{2}{*}{ Tigecycline } & S & 56 & 38 & 94 \\
\hline & $\mathrm{R} / \mathrm{I}$ & 17 & 69 & 86 \\
\hline \multicolumn{2}{|c|}{ Total } & 73 & 107 & 180 \\
\hline \multicolumn{5}{|c|}{$\begin{array}{l}\text { Table 4. Relative Susceptibilities of Isolates } \\
\text { towards Tigecycline and Minocycline }\end{array}$} \\
\hline
\end{tabular}

\begin{tabular}{|cccc|}
\hline Isolates & $\begin{array}{c}\text { Sensitive } \\
\text { Concordance }\end{array}$ & $\begin{array}{c}\text { Cohen Kappa } \\
\text { Value }\end{array}$ & $\begin{array}{c}\text { Concordance } \\
\text { Acinetobacter species }\end{array}$ \\
$31.1 \%(180)$ & 0.394 & Fair \\
\hline Table 5. Degree of Sensitive Concordance in the Susceptibility to \\
Minocycline and Tigecycline against Acinetobacter species
\end{tabular}

$21.1 \%$ isolates were resistant / intermediate to minocycline but sensitive to tigecycline. Only $9.4 \%$ isolates which were resistant to tigecycline were sensitive to minocycline. The data is shown in Table 4 and 5 . The sensitive concordance was $31.1 \%$, which indicated fair agreement statistically.

\section{DISCUSSION}

Carbapenems are considered as the drug of choice for Acinetobacter baumannii infections. In this study, high rate of carbapenem resistant Acinetobacter species was seen $(80.7$ \%). In a study by Devrajan V et al. in the year 2014, $75.8 \%$ of the Acinetobacter isolates studied were carbapenem resistant. ${ }^{11}$ In the study by Odsbu I et al from Nashik district in Western India, the proportion of Acinetobacter species resistant to carbapenems ranged from $81.6 \%$ to $88.3 \%$ between 2011 and 2014.12 A study at Varanasi showed that 93 $\%$ of the Acinetobacter species studied were blaOXA23 producers, which confers high level resistance towards carbapenems. $^{13}$ Mechanism of resistance for all the carbapenem antibiotics could be due to the production of carbapenemases, though the detection of the mechanisms of resistance was not attempted in this study. It was observed that $89.3 \%$ of the isolates were resistant to all the 3 carbapenem antibiotics, namely imipenem, meropenem and doripenem. Resistance to 2 or a single carbapenem may be due to other mechanisms like impermeability, loss of outer membrane proteins. ${ }^{14}$ In a study by Coskun USS et al. $100 \%$ of the study isolates of Acinetobacter baumanii were resistant to imipenem with blaOXA-23 gene in all of them. Also, $45.8 \%$ resistance rates towards tigecycline was noted among the 96 isolates studied. 15

In this study, isolates were found to be more sensitive towards tigecycline than minocycline. $54.1 \%$ isolates were sensitive to tigecycline while sensitivity towards minocycline was $40.5 \%$. Arroyo La et al. tested 150 isolates of A. baumannii and found that tigecycline MICs were 3 dilutions lower than minocycline MICs. ${ }^{16}$ Some studies have contrasting results. Deng $\mathrm{M}$ et al showed that high percentage of Acinetobacter baumannii were still susceptible to minocycline; $40.6 \%$ of the tigecycline non-susceptible isolates were susceptible to minocycline. Lashinsky JN et al reviewed various studies and concluded that minocycline was the second active agent after colistin. ${ }^{17}$ The most important mechanism for acquiring resistance towards tetracycline is by efflux pumps. The genes coding for TetB efflux pumps are effective in transporting out minocycline. Lomoskaya et al. showed that presence of TetB had a $93 \%$ positive predictive value for minocycline resistance. Only 11 / 165 (6.7 \%) were susceptible when Tet B was present. ${ }^{18}$ Tigecycline has the ability to overcome most of the efflux pumps. Many other mechanisms of resistance towards tigecycline can be seen in Acinetobacter species. Resistance nodulation division (RND) type efflux pumps like AdeABC transport drug into the extracellular space. Expression of these RND pumps is associated with higher MICs and increased resistance to tigecycline in vitro. RND pumps do not act on minocycline. MICs for Acinetobacter isolates may increase only after brief exposure to tigecycline during therapy, within a matter of few weeks. This is due to upregulation and over-expression of RND pumps while on tigecycline therapy. ${ }^{19}$

Distribution of isolates based on the samples did not show much of a difference with isolates from blood and exudates showing $>80 \%$ carbapenem resistance. $52 \%$ of the urine isolates were carbapenem resistant. This may include more of community acquired urinary tract infections; which are generally more sensitive towards antibiotics. Sensitivity rates towards both tigecycline and minocycline increased as the 
number of carbapenem drugs to which they were resistant decreased, showing possibly carbapenemase producing Acinetobacter developing resistance towards tigecycline and minocycline. This may be because hospital strains of Acinetobacter especially in Intensive Care Units may have already been exposed to tigecycline / minocycline and acquired resistance. Further, isolates from blood were more resistant to tigecycline and minocycline compared to the isolates from other sites.

The sensitive concordance in susceptibility to minocycline and tigecycline was $31.1 \%$. This was similar to the study by Warrier AR et al; with $33.3 \%$ in their study. ${ }^{10}$ Also $50 \%$ of the Acinetobacter species studied were sensitive to minocycline. This study showed $21 \%$ of the isolates with resistant or intermediate susceptibility towards minocycline showed tigecycline susceptible. Tigecycline appears to be a better agent for treatment of morbid infections. Tigecycline provides higher concentration in tissues; hence it is indicated in the treatment of complicated skin / skin structure and intraabdominal infections. Post antibiotic effect is more for tigecycline than minocycline (0.6 - 2 hours). ${ }^{6}$ But, action of minocycline in vivo could have an upper hand as it has been demonstrated to have better pharmacodynamic and pharmacokinetic action compared to tigecycline. Minocycline also reaches good tissue concentrations and is easy to switch from intravenous to oral formulations.

Monotherapy with tigecycline / minocycline must be avoided. In a retrospective study of 70 critically ill patients with Multi drug resistance (MDR) Acinetobacter baumannii pneumonia; with 30 patients receiving tigecycline based therapy and 40 patients receiving colistin based therapy; the clinical success rate was found to be similar. However, number of mortalities were lower in patients who received combination therapy. ${ }^{20}$ Liang $\mathrm{W}$ et al showed that combinations of colistin with meropenem, rifampicin or minocycline had synergistic activity. Also minocycline and meropenem combination showed bactericidal activity in vitro against extensive drug resistant Acinetobacter baumannii strains. Only colistin showed bactericidal activity when tested alone. ${ }^{21}$ Shin JR et al studied the clinical and microbial efficacy of tigecycline for multidrug resistant Acinetobacter baumannii infections. They found that though microbial eradication rate was high and clinical success rate was low, the utility of tigecycline could not be overlooked. They opined that combination therapy provided better clinical and microbial success rate and that other than colistin, tigecycline is the only drug active against carbapenem resistant Acinetobacter baumannii. Therefore, utility of tigecycline cannot be overlooked.22

In a study from north-eastern India, $79.2 \%$ of the study isolates were found to exhibit high level aminoglycoside resistance with an MIC of $>=512 \mu \mathrm{g} / \mathrm{ml}$. Among them $83.8 \%$ had acquired aminoglycoside resistant genes with co-existent Extended spectrum beta- lactamases (ESBL) and metallo betalactamase (MBL) genes. For such cases, polymyxins, tigecyclines, minocycline could be lifesaving. Even then, monotherapy with these drugs should be avoided, as there is risk of rapid development of resistance to these drugs by such pan drug resistant isolates. Hence, the authors concluded that the use of these drugs would be justified in group of critically ill patients in the ICUs. ${ }^{3}$

\section{CONCLUSIONS}

Minocycline and tigecycline are effective against carbapenem resistant Acinetobacter species. Tigecycline has also been effective against few isolates which were resistant to minocycline. This result suggests that tigecycline can be considered as a therapeutic agent for the treatment of multidrug resistant Acinetobacter which are otherwise difficult to inhibit using other antibiotics. Some isolates demonstrating resistance against tigecycline and minocycline is a matter of great concern. It emphasises the need for judicious use of these lifesaving antibiotics and strong infection control measures. To combat this problem; WHO recommends five infection prevention strategies; namely hand hygiene, surveillance for carbapenem resistant bugs, contact precautions, environmental cleaning and patient isolation in single room or by cohorting. These measures have to be boosted by a strong system for monitoring, auditing of strategies and feedback to health care workers and decision makers. ${ }^{23}$

Data sharing statement provided by the authors is available with the full text of this article at jemds.com.

Financial or other competing interests: None.

Disclosure forms provided by the authors are available with the full text of this article at jemds.com.

\section{REFERENCES}

[1] Alsan M, Klompas M. Acinetobacter baumannii: an emerging and important pathogen. J Clin Outcomes Manag 2010;17(8):363-9.

[2] Hsu LY, Apisarnthanarak A, Khan E, et al. Carbapenemresistant Acinetobacter baumannii and enterobacteriaceae in South and Southeast Asia. Clin Microbiol Rev 2017;30(1):1-22.

[3] Upadhyay S, Khyriem AB, Bhattacharya P, et al. High-level aminoglycoside resistance in Acinetobacter baumannii recovered from intensive care unit patients in Northeastern India. Indian J Med Microbiol 2018;36(1):43-8.

[4] Isler B, Doi Y, Bonomo RA, et al. New treatment options against carbapenem-resistant Acinetobacter baumannii infections. Antimicrob Agents Chemother 2018;63(1):e01110-18.

[5] Lashinsky JN, Henig O, Pogue JM, et al. Minocycline for the treatment of multidrug and extensively drug-resistant $A$. baumannii: a review. Infect Dis Ther 2017;6(2):199-211.

[6] Pankey GA. Tigecycline. J Antimicrob Chemother 2005;56(3):470-80.

[7] CLSI. Performance Standards for Antimicrobial Susceptibility Testing. 28 th edn. CLSI supplement M100. Wayne, PA: Clinical and Laboratory Standards Institute 2018.

[8] European Committee on Antimicrobial Susceptibility Testing (EUCAST). Breakpoint tables for interpretation of MICs and zone diameters. Version 8.0. Valid from 01 January, $2018 . \quad$ p. $\quad 8$. http://www.eucast.org/clinical_breakpoints/. 
[9] Marchaim D, Pogue JM, Tzuman 0, et al. Major variation in MICs of tigecycline in gram negative bacilli as a function of testing method. J Clin Microbiol 2014;52(5):1617-21.

[10] Warrier AR, Babu R. Susceptibility of minocycline against carbapenem resistant gram negative bacilli. J Microbiol Infect Dis 2018;8(4):140-6.

[11] Devarajan V, Bansal N, Ghafur KA. Comparative analysis of tigecycline and minocycline susceptibility among gramnegative isolates. J Contemp Clin Pract 2018;4(1):8-12.

[12] Odsbu I, Khedkar S, Khedkar U, et al. High proportions of multidrug-resistant acinetobacter spp. Isolates in a district in Western India: a four-year antibiotic susceptibility study of clinical isolates. Int J Environ Res Public Health 2018;15(1):153-66.

[13] Banerjee T, Mishra A, Das A, et al. High prevalence and endemicity of multidrug resistant acinetobacter spp. in intensive care unit of a tertiary care hospital, Varanasi, India. Journal of Pathogens 2018;2018:9129083.

[14] Poirel L, Nordmann P. Carbapenem resistance in Acinetobacter baumannii: mechanisms and epidemiology. Clin Microbiol Infect 2006;12(9):826-36.

[15] Coskun USS, Caliskan E, Cicek AC, et al. $\beta$-lactamase genes in carbapenem resistance Acinetobacter baumannii isolates from a Turkish University Hospital. J Infect Dev Ctries 2019;13(1):50-5.

[16] Arroyo LA, Matros I, Gonzalez V, et al. In vitro activities of tigecycline, minocycline and colistin-tigecycline combination against multi- and pandrug resistant clinical isolates of Acinetobacter baumannii group. Antimicrob Agents Chemother 2009;53(3):1295-6.
[17] Deng M, Zhu MH, Li JJ, et al. Molecular epidemiology and mechanisms of tigecycline resistance in clinical isolates of Acinetobacter baumannii from a Chinese University Hospital. Antimicrob Agents Chemother 2014;58(1):297303.

[18] Lomovskaya O, Sun D, Rubio-Aparicio D, et al. Absence of TetB identifies minocycline-susceptible isolates of Acinetobacter baumannii. Int J Antimicrob Agents 2018;52(3):404-6.

[19] Reid GE, Grim SA, Aldeza CA, et al. Rapid development of Acinetobacter baumannii resistance to tigecycline. Pharmacotherapy 2007;27(8):1198-201.

[20] Kim WY, Moon JY, Huh JW, et al. Comparable efficacy of tigecycline versus colistin therapy for multidrug resistant and extensively drug-resistant Acinetobacter baumannii pneumonia in critically Ill patients. PLoS One 2016;11(3):e0150642.

[21] Liang W, Liu XF, Huang J, et al. Activities of colistin- and minocycline-based combinations against extensive drug resistant Acinetobacter baumannii isolates from intensive care unit patients. BMC Infect Dis 2011;11:109.

[22] Shin JA, Chang YS, Kim HJ, et al. Clinical outcomes of tigecycline in the treatment of multidrug-resistant Acinetobacter baumannii infection. Yonsei Med J 2012;53(5):974-84.

[23] WHO. Guidelines for the prevention and control of carbapenem-resistant enterobacteriaceae, Acinetobacter baumannii and pseudomonas aeruginosa in health care facilities. Geneva: World Health Organization 2017. 